Exp. Anim. 49(2), 91-96, 2000

\title{
Partial Hepatectomy of Marmoset: Clinical and Pathological Effects and Utility in Microsomal Enzyme Analysis
}

\author{
Yoshimasa KURATA'), Futoshi MAKINODAN ${ }^{1)}$, J unko MATSUMOTO1), \\ Naoto TOYOTA ${ }^{1)}$, and Keisuke TANAKA ${ }^{2)}$
}

\begin{abstract}
1)Mitsubishi Chemical Safety Institute Ltd., 14 Sunayama, Hasaki-machi, Kashima-gun, Ibaraki, 314-0255 and ${ }^{2) M i t s u b i s h i ~ C h e m i c a l ~ C o r p o r a t i o n, ~ Y o k o h a m a ~ R e s e a r c h ~ C e n t e r, ~}$ 1000 Kamoshida, Aoba-ku, Yokohama-shi, Kanagawa, 227-0033, Japan
\end{abstract}

\begin{abstract}
Liver biopsy based on a partial hepatectomy technique (shearing) was performed in 10 common marmosets (Callithrix jacchus). This is a preliminary study to evaluate the effects of drugs on hepatic microsomal enzymes: cytochrome $P-450$ and $T_{4}$ uridine diphosphate glucuronyl transferase ( $\left.T_{4}-U D P G T\right)$, by comparing post-treatment values with pre-treatment values individually with a limited number of animals. The effects of the biopsy on clinical findings and liver pathology were evaluated during the first 5 postsurgical weeks. Although the plasma aspartate aminotransferase (AST) activities tended to decrease from 1 to 4 weeks post-surgery, no abnormality was noted in clinical sign, body weight, the hematocrit value or other blood chemical values. At necropsy, adhesion of the sheared site of the liver to the parietal peritoneum or the small intestine was evident in 2 of the 4 marmosets. Microscopic examination revealed focal fibrosis in the liver, but it was localized around the sheared site. Based on the above results, it was concluded that liver biopsy must be performed more than one month before administration of the drug to be tested. The biopsy samples and the whole liver samples obtained at autopsy were subjected to analysis of microsomal protein content, cytochrome P-450 content and $T_{4^{-}}$ UDPGT activity. In comparison with the values from the whole liver samples, those from the biopsy samples showed no significant difference. Furthermore, there was a significant correlation rather than difference between matched values. This suggested that partial hepatectomy is a useful method for obtaining pretreatment values in liver biochemistry to evaluate the effects of drug-treatment in individual animals.
\end{abstract}

Key words: common marmoset, cytochrome P-450, partial hepatectomy, systemic effect, T4-UDPGT

(Received 23 June 1999 / Accepted 19 November 1999)

Address corresponding: Y. Kurata, Mitsubishi Chemical Safety Institute Ltd., 14 Sunayama, Hasaki-machi, Kashima-gun, Ibaraki, 314-0255, Japan 


\section{Introduction}

Biochemical analysis of the liver is valuable in toxicological assessment of drugs, as this organ is frequently a target of toxicity and a highly active site of metabolism, but these data are usually obtained only at postmortem examination, and the pretreatment state is unknown. Furthermore, in toxicological studies in primates including marmosets, animals with large variations in age, body weight, and clinical examinations constitute one dosage group. Liver biopsy is thought to be an effective method for examining the pretreatment histological and biochemical state of the organ, and for evaluating drug effects with only a limited number of animals by comparing post-treatment state with pre-treatment state individually.

Partial hepatectomy is useful for obtaining large tissue samples, which are required in biochemical analysis, tissue culture and for other purposes $[3,6,7]$. On the other hand, the systemic effect of the general anesthesia, bleeding from the surgical site, and injury to the liver would be greater than in closed needle biopsy. In this study, the effects on clinical findings and liver pathology and usefulness in biochemical analysis of partial hepatectomy were determined in the marmoset.

Liver samples were also used for the analysis of $\mathrm{P} 450$ content and $\mathrm{T}_{4}$-UDPGT activity. It has been reported that an enhancement of thyroxin metabolism in the liver can induce thyroid hyperactivity or neoplasia, but there are marked species differences in this phenomenon [5, 10]. This was conducted as a preliminary study to determine the correlation between microsomal enzyme induction and thyroid function in the marmoset.

\section{Materials and Methods}

\section{Animals and husbandry}

Ten male common marmosets (Callithrix jacchus) used in this study were purchased from CLEA Japan Inc. (Tokyo, Japan) or bred in our laboratory. The body weight and age at the liver biopsy ranged from 265 to $405 \mathrm{~g}$ and from 17 to 38 months, respectively. The marmosets were housed individually in stainless steel wire-mesh cages $(300 \mathrm{~W} \times 600 \mathrm{D} \times 650 \mathrm{H} \mathrm{mm})$, under conditions of constant of room temperature (26 \pm $2^{\circ} \mathrm{C}$ ) and relative humidity $(55 \pm 15 \%)$, with air exchanged 15 times an hour and a light and dark cycle of
$12 \mathrm{hr}$ (with light from 8:00 to 20:00). They were fed a mixture of pelleted diet for new world monkeys (SPS, Oriental Yeast Co. Ltd., Tokyo, Japan), ascorbic acid, and honey, each morning. Water was provided ad libitum from a water bottle with tap water which was passed through a $5 \mu \mathrm{m}$ filter and irradiated with UV light. The animals were treated with appropriate care and respect, according to the Guidelines for Animal Experimentation of our Laboratory, Mitsubishi Chemical Safety Institute Ltd.

\section{Liver biopsy procedure}

Following general anesthesia with sodium pentobarbital (30 mg/kg, ip), the marmoset was held on its back. The surgical site was clipped free of hair and disinfected with povidone-iodine and $70 \%$ ethanol. An incision about $2 \mathrm{~cm}$ in length in the skin and the linea alba was made just under the ensiform cartilage. The right lateral lobe of the liver was exteriorized by pressing the abdomen with the fingers, and held loosely with a pair of tweezers with tips wrapped in moist gauze, and the wedge of the lobe thus formed was sheared with a pair of surgical scissors (Fig. 1a). A collagen sheet (AVITENE, Zeria Shinyaku Co. Ltd., Tokyo, Japan) was used for hemostasis by placing it on the sheared site soon after shearing (Fig. 1b). A few minutes later, the excess hemostatic material was washed off with saline. The suture site was disinfected with povidone-iodine once a day for about a week until removal of the sutures.

\section{Clinical examination}

Before surgery and once a week after surgery for 5 weeks, hematological and blood biochemical tests were done in four marmosets. A blood sample (approximately $1.5 \mathrm{ml}$ ) was withdrawn from the femoral vein and put into a heparinized tube. After measuring of hematocrit, plasma separated by centrifugation was used to measure of alanine aminotransferase (ALT), aspartate aminotransferase (AST), lactate dehydrogenase (LDH), alkaline phosphatase (ALP), $\gamma$-glutamyltranspeptitase $(\gamma$ GTP), total protein (TP), and total bilirubin (T-BIL) with an auto analyzer (736-10, HITACHI, Ltd., Tokyo, Japan).

\section{Pathological examination}

Five weeks after surgery, four marmosets were killed 


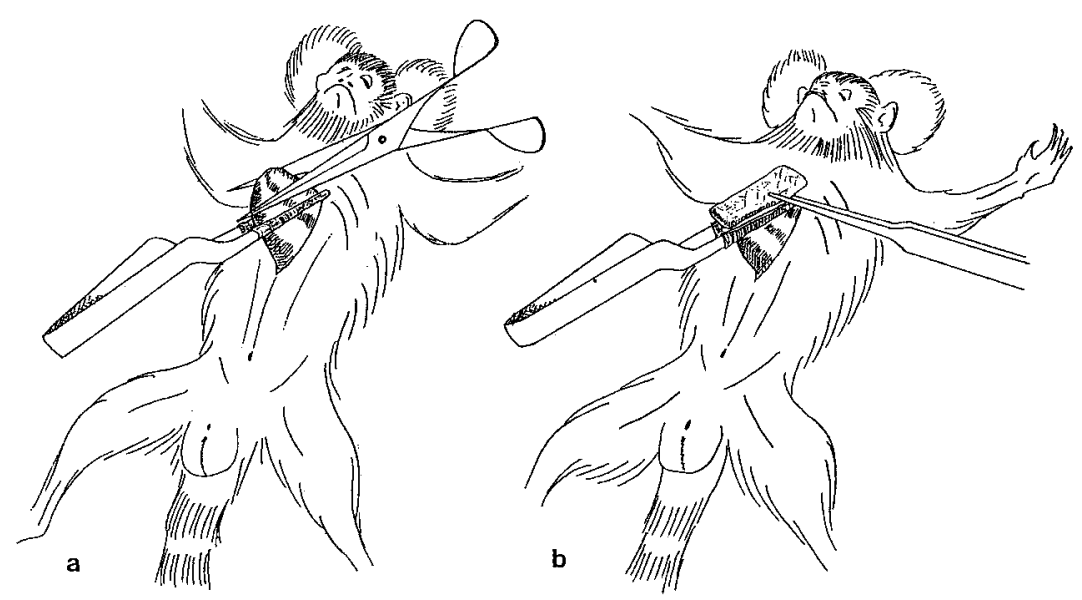

Fig. 1. Liver biopsy procedure based on partial hepatectomy. Drawings show (a) method for holding and shearing the liver lobe and (b) hemostasis of the sheared site.

after the clinical examination described above by exsanguination of the abdominal aorta under sodium pentobarbital anesthesia and subjected to necropsy. The liver and other organs were removed and fixed in $10 \%$ buffered neutral formalin, and then embedded in paraffin blocks. Light microscopic examination of the liver was done on sections stained with hematoxylin and eosin.

\section{Analysis of Cytochrome P-450 content and $T_{4}-U D P G T$ - activity}

Liver homogenates (20\%) were prepared with $0.25 \mathrm{M}$ sucrose, $10 \mathrm{mM}$ HEPES and $1 \mathrm{mM}$ dithiothreitol solution ( $\mathrm{pH}$ 7.0) for ten marmosets including six animals used as controls in 4 weeks toxicological studies and four animals 5 weeks after hepatectomy with a PotterElvehjem type motor driven glass and Teflon homogenizer. After the centrifugation of whole homogenates at $10,000 \times \mathrm{g}$ for $20 \mathrm{~min}$, microsomal fractions were obtained by centrifugation of the supernatants at $100,000 \times \mathrm{g}$ for $60 \mathrm{~min}$ and diluted with $0.1 \mathrm{M}$ potassium phosphate buffer (pH 7.4) containing 2 mM EDTA and $1 \mathrm{mM}$ dithiothreitol. Microsomal protein contents were measured according to Lowry's method [4], and cytochrome P-450 contents were measured according to Oumura's method [9]. Uridine diphosphate glucuronyltransferase (UDPGT) activities were measured according to Beetstra's method [1] using L-thyroxin $\left(\mathrm{T}_{4}\right)$ as a substrate.

\section{Statistics}

Body weight, hematocrit, and blood chemical parameters were analyzed by two-way analysis of variance. When the difference was found to be significant, each postoperative value was compared by paired $t$-test against the preoperative value. Liver enzymes in biopsy specimens and whole liver samples were analyzed by paired $t$-test and Pearson's correlation coefficient.

\section{Results}

\section{Weight of biopsy samples}

The weight of the liver sample obtained by this biopsy procedure ranged from 0.24 to $0.59 \mathrm{~g}$, corresponding to 1.4 to $4.9 \%$ of the whole liver weight at necropsy.

\section{Body weight change and clinical findings}

During the 5-week postoperative period, no abnormality was observed in clinical signs or body weight change. There was a significant decrease in the AST value at 1,3 , and 4 weeks after surgery compared with the preoperative value, but no abnormality was observed during this period in any other blood chemical quantities, such as ALT, LDH, ALP, $\gamma$-GTP, TP, and T-BIL and the hematocrit value in any of the four animals (Fig. 2).

\section{Necropsy and histopathological findings}

Necropsy revealed adhesion of the sheared site of the liver to the abdominal wall or small intestine in two of the four marmosets. Histologically, fibrosis accom- 

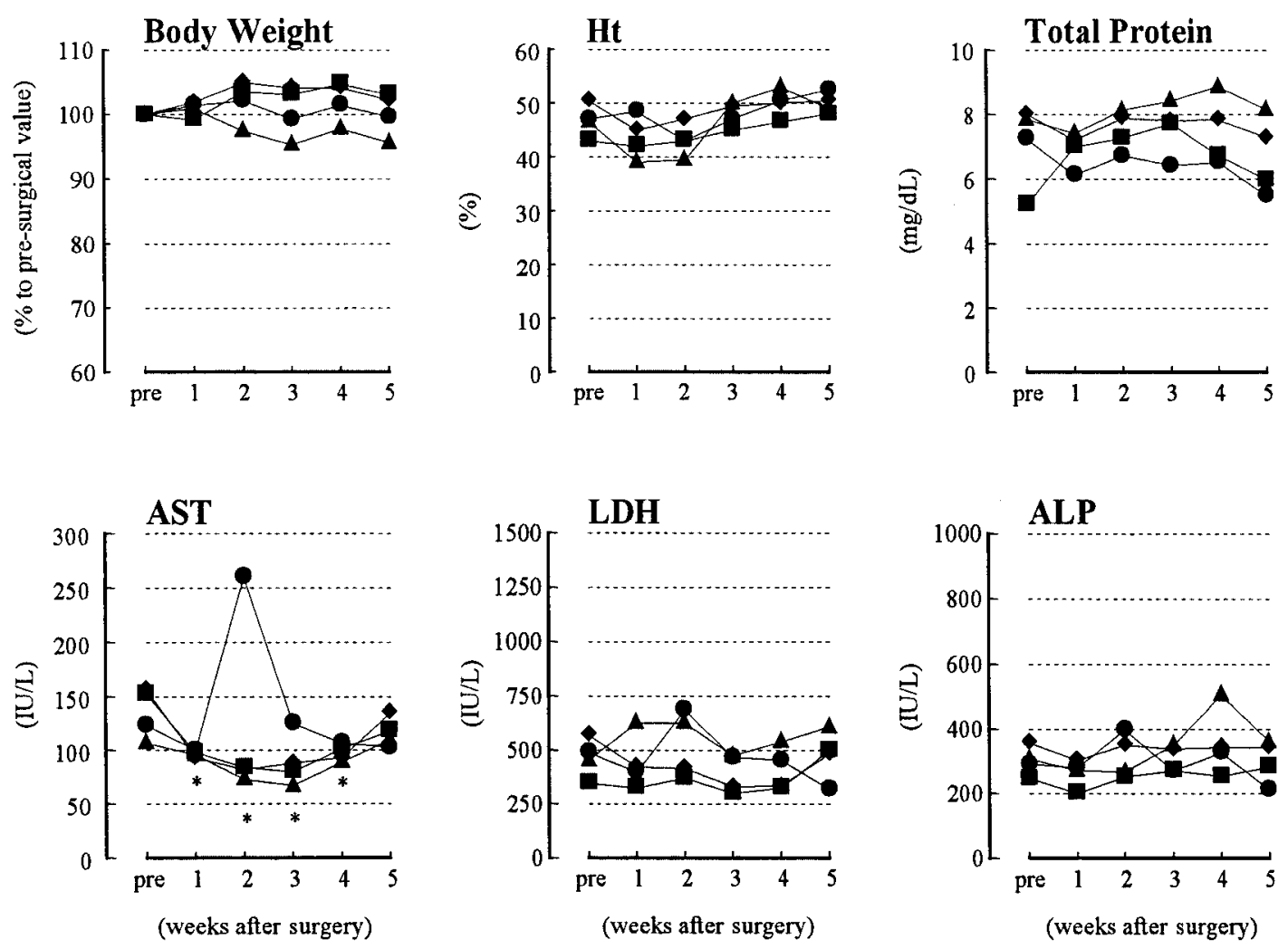

Fig. 2. Body weight, hematocrit and blood chemical changes in marmosets after liver biopsy. Data are shown individually. As one animal (indicated with a ) had a temporary increase in the AST value 2 weeks after the operation, it was excluded, and AST values significantly $(*, p<0.05)$ decreased.

Table 1. Macro- and Microscopic Findings in Marmoset Livers 5 Weeks after Liver Biopsy

\begin{tabular}{|c|c|c|c|c|}
\hline \multirow{2}{*}{$\begin{array}{l}\text { Examinations } \\
\text { Findings }\end{array}$} & \multicolumn{4}{|c|}{ Marmoset No. } \\
\hline & 1 & 2 & 3 & 4 \\
\hline \multicolumn{5}{|l|}{ Macroscopic examination } \\
\hline Adhesion to peritoneum or small intestine & $\mathrm{P}$ a) & & & $\mathrm{P}$ \\
\hline \multicolumn{5}{|l|}{ Microscopic examination } \\
\hline Focal fibrosis at sheared site & + b) & + & + & + \\
\hline Extramedullary hematopoiesis & & & + & \\
\hline Fatty droplets in Kuffer cells & & & & + \\
\hline Central accumlation of glycogen & + & & & \\
\hline
\end{tabular}

a): present, b): slight.

panied by slight infiltration of inflammatory cells was observed around the remnant hemostatic material on the sheared site of the liver in all four marmosets (Table 1). In addition, increases in extramedullary hematopoiesis, fatty infiltration in Kupffer cells, and centrilobular accumulation of glycogen were observed in one marmoset each; but such findings are often present spontaneously in this species.

\section{Cytochrome P-450 content and $T_{4}-U D P G T$ activity}

For the biopsy specimens, the microsomal protein content, cytochrome P-450 content, and $\mathrm{T}_{4}$-UDPGT ac- 
Microsomal protein

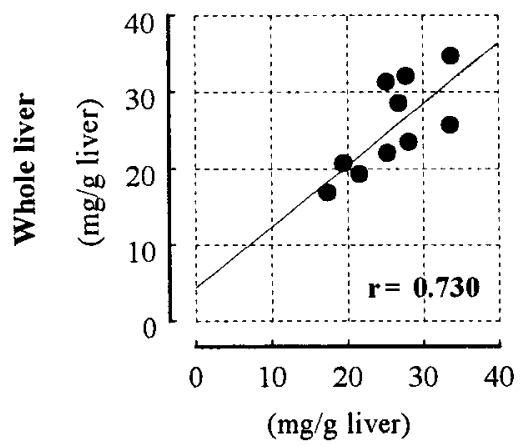

Biopsy specimen
Cytochrome P-450

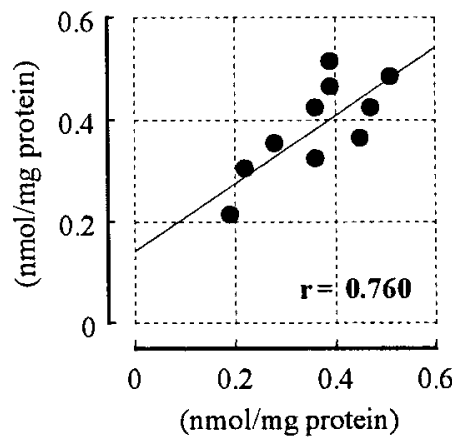

Biopsy specimen
T4-UDPGT

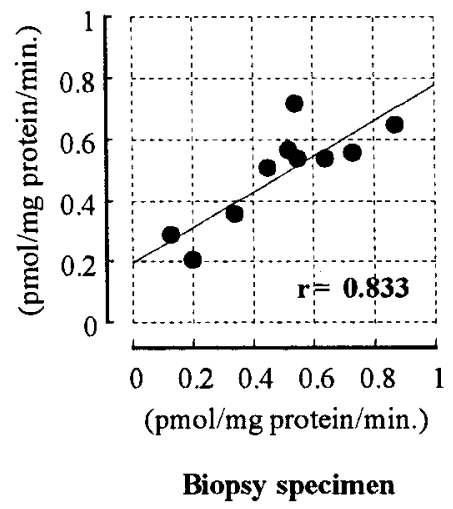

Fig. 3. Correlation $(n=10)$ between values obtained in biopsy specimens and whole liver samples 5 or 9 weeks later.

tivity ranged from 17.4 to $33.8 \mathrm{mg} / \mathrm{g}$ liver, 0.19 to 0.51 $\mathrm{nmol} / \mathrm{mg}$ protein, and 0.13 to $0.87 \mathrm{pmol} / \mathrm{mg}$ protein/ min, respectively. These values for the entire liver ranged from 16.6 to $34.5 \mathrm{mg} / \mathrm{g}$ liver, 0.21 to $0.51 \mathrm{nmol} /$ $\mathrm{mg}$ protein, and 0.20 to $0.71 \mathrm{pmol} / \mathrm{mg}$ protein $/ \mathrm{min}$, respectively. No significant difference was observed between the biopsy and whole liver samples in any of these values. The biopsy values were significantly correlated with the autopsy values ( $\mathrm{r}=0.730$ for microsomal protein content, 0.760 for cytochrome P-450 content, and 0.833 for $\mathrm{T}_{4}$-UDPGT activity) (Fig. 3).

\section{Discussion}

The effects of partial lobectomy on clinical findings and liver pathology were determined for potential application of this technique to toxicological studies in the marmosets. During and after the biopsy procedure, very little bleeding from the incision in the skin and linea alba was noted, and the efficacy of the hemostatic material applied to the sheared site of the liver was indicated by the slight decline in the hematocrit value found during the post-surgical period. This study indicated that no other hemostatic treatment was required, and the simple surgical procedure could be completed within only 10 or $15 \mathrm{~min}$.

The blood chemical examination revealed a decline in the AST value 1 to 4 weeks after the operation. It was not considered that this slight change would be of clinical significance, because the blood level of the enzyme usually increases just after organs are injured.
One animal had a temporary but significant increase in the blood AST level at 2 weeks after the operation, but no relationship was observed between this change and pathological findings or sample volumes removed at biopsy. Previous studies have revealed changes in some clinical quantities within the first week after surgery; for instance, an increase in the ALT value was observed in the chimpanzee after partial lobectomy of the liver [7], but in the present study no change in other indicators of liver injury or functional disorder, including plasma ALT, LDH, ALP, $\gamma$-GTP, TP, LAP, and T-BIL values, was observed during the postoperative period. Pathological examination was performed 5 weeks after the operation, when it was thought that no clinical effect of the biopsy procedure remained. The histological changes observed at this time suggested local response to the residual hemostatics and recovery from the surgical injury. There was no evidence of extension of inflammatory lesion or parenchymal disorder. These findings suggested that clinical and pathological effects of drug-treatment on the liver can be assessed with no difficulty by this procedure. Furthermore, it was indicated that the optimum time for liver biopsy by this procedure is approximately one month or more before commencement of drug treatment.

No obvious difference was observed between the whole-liver and the biopsy specimen in microsomal protein content, cytochrome P-450 content, or $\mathrm{T}_{4}$-UDPGT activity, and significant correlations were observed in each matched value in spite of large individual variations, especially in P-450 content and $\mathrm{T}_{4}$-UDPGT activity. 
This procedure is therefore considered to be useful in the evaluation of drug effects by comparison with the pretreatment values in the same animal as an alternative to the mean values in control animals, and to be effective especially in a study of only a limited number of animals with large individual variations. But it also remains necessary to examine the differences between biopsy specimens and whole liver in other liver function quantities, such as P450 isoformes or phase II enzymes,.

Miller et al. [8] determined hepatic monooxigenase activities in rhesus monkeys with needle biopsy specimens, and compared them with the activities of whole liver homogenates. Bottenus et al. [2] also established a preparation method for hepatic mitochondria and microsomes from needle biopsy specimen in African green monkeys. The closed needle biopsy procedure is therefore thought to be effective for continual monitoring of liver functions, but there were some differences between biopsy specimens and whole liver samples in protein content and enzyme activities, which may be partly due to the small sample size ( $<30$ or $\sim 100 \mathrm{mg}$ ). The partial hepatectomy procedure allows a comparatively large amount of tissue to be obtained even in small-size animals like marmosets (250-500 g of body weight), whereas it may be difficult to obtain sufficiently large samples by the needle biopsy procedure.

\section{Acknowledgments}

We thank Dr. Minoru Tsuchitani for valuable discussions and Mr. Takeshi Kawasuso for assistance in preparing the manuscript.

\section{References}

1. Beetstra, J.B., Van Engelen, J.G. M., Karels, P., Van der Hoe, H.J., De Jong, M., Docter, R., Krenning, E.P., Hennemann, G., Brouwer, A., and Visser, T.J. 1991. Thyroxine and 3,3',5-triiodothyronine are glucuronidated in rat liver by different uridinediphosphateglucuronyltransferases. Endocrinology 128: 741-746.

2. Bottenus, R.E. and Cunningham, C.C. 1982. Preparation of coupled mitochondria and microsomes from liver biopsy samples of african green monkeys. Prep. Biochem. 12: 197207.

3. Eichberg, J.W. 1985. Liver wedge biopsy in nonhuman primates. J. Med. Primatol. 14: 165-168.

4. Lowry, O.H., Rosebrough, N.J., Farr, A.A., and Randall, R. J. 1951. Protein measurement with the folin phenol reagent. J. Biol. chem. 193: 265-275.

5. McClain, R.M. 1992. Thyroid gland neoplasia: Nongenotoxic mechanisms. Toxicol. Lett. 64/65: 397-408.

6. Michael, R.T. and Robert, C.D. 1991. Partial lobectomy via a ligature fracture technique: A method of multiple hepatic biopsies in nonhuman primates. Lab. Animal Sci. 41: 476-480.

7. Nolan, T.E. and Conti, P.A. 1980. Liver wedge biopsy in chimpanzees (pan troglodytes) using an automatic sampling device. Lab. Animal Sci. 30: 578-580.

8. Miller, J.L., Gee, S.J., Krieger, R.I., and Ruebner, B.H. 1978. Closed needle liver biopsy for assessment of monooxygenase activity in rhesus monkeys (Macaca mulatta). J. Med. Primatol. 7: 1-7.

9. Oumura, T. and Sato, R. 1964. The carbon monoxidebinding pigment of liver microsomes. J. Biol. Chem. 239: 2370-2378.

10. Zbinden, G. 1987. Assessment of hyperplastic and neoplastic lesions of the thyroid gland. Trends Pharmacol. Sci. 8: 511-514. 